\section{0-01. miR-142-3p as a biomar- ker of blastocyst implantation failure - a pilot study}

Edson Borges Jr. ${ }^{1,2}$, Amanda Souza Setti, 1,2, Daniela P.A. F. Braga ${ }^{1,2,3}$, Murilo V Geraldo ${ }^{4}$, Rita de Cássia S Figueira $^{1}$, Assumpto Iaconelli Jr' ${ }^{1}$

\author{
${ }^{1}$ Fertility Medical Group - São Paulo/SP - Brazil \\ ${ }^{2}$ Instituto Sapientiae - Centro de Estudos e Pesquisa em \\ Reprodução Assistida - São Paulo/SP - Brazil \\ ${ }^{3}$ Disciplina de Urologia, Área de Reprodução Humana, \\ Departamento de Cirurgia, Universidade Federal de São \\ Paulo/SP - Brazil \\ ${ }^{4}$ Departamento de Biologia Estrutural e Funcional, Instituto \\ de Biologia, Universidade Estadual de Campinas - UNICAMP \\ - SP. Brazil
}

Objective: To investigate if microRNAs (miRNAs) detected in the culture media of individual in vitro produced embryos could be potential biomarkers of embryo implantation.

Methods: Culture media samples from 36 embryos, derived from patients undergoing intracytoplasmic sperm injection (ICSI) in a private university-affiliated IVF center, were collected between January/2015 and November/2015. Samples were collected on day three and embryo transfers were performed on day five and all embryos reached the blastocyst stage. Samples were split into groups according to the embryo implantation result: Positive-ImplantationGroup $(n=18)$ or Negative-Implantation-Group $(n=18)$. For the first analysis, samples were pooled in three sets for each group (6-7 spent media per pool). MicroRNAs were extracted from spent media and CDNA was synthesized. C. elegans miR-39 was used as RNA spike-in to normalize the gene expression analysis. The expression of microRNAs into the spent media from the Positive-Implantation-Group was compared with those from the Negative-Implantation-Group. A set of seven miRNAs (miR-21, miR-142-3p, miR-19b, miR-92a, miR-20b, miR-125a and miR148a) selected according with the literature, was tested. To check whether miRNAs could be detected in individual samples of culture media, in a second analysis, ten more samples were tested for miR-21 and miR-142-3p.

Results: From the sevens tested miRNAs, a significant increased expression of miR-142-3p could be noted in the Negative-Implantation-Group $(P<0.001)$. For other three miRNAs (miR-21, miR-19b and miR-92a) a difference in expression was observed, however it did not reach a statistical significance. In addition, when ten non-redundant samples were tested to check if miRNAs could be detected in individual samples of culture media, the highly specific amplification of mature miRNAs, including miR$142-3 p$, could be noted.

Conclusions: Our findings suggest that miR-142-3p, previously described as a tumor suppressor and cell cycle inhibitor may be a potential biomarker of blastocyst implantation failure. The identification of miRNAs on individual culture media samples offers unique opportunities for non-invasive early diagnosis of blastocyst implantation.

\section{0-02. Non-invasive prediction of blastocyst implantation, ongoing pregnancy and live birth, by mass spectrometry lipid fingerprinting}

Edson Borges Jr.1,2, Daniela P.A.F. Braga ${ }^{1,2,3}$, Amanda Souza Setti ${ }^{1,2}$, Daniela A. Montanni ${ }^{3}$, Elaine Cristina Cabral ${ }^{4}$, Marcos N. Eberlin ${ }^{4}$, Edson G. Lo Turco ${ }^{3}$, Assumpto Iaconelli Jr ${ }^{1}$

${ }^{1}$ Fertility Medical Group - São Paulo/SP - Brazil

${ }^{2}$ Instituto Sapientiae - Centro de Estudos e Pesquisa em Reprodução Assistida - São Paulo/SP - Brazil

${ }^{3}$ Disciplina de Urologia, Área de Reprodução Humana, Departamento de Cirurgia, Universidade Federal de São Paulo/SP - Brazil

${ }^{4}$ Laboratório ThoMSon de Espectrometria de Massas Instituto de Química - UNICAMP

Objective: To identify lipid markers of blastocyst implantation and ongoing pregnancy by day three culture medium mass spectrometry (MS) fingerprinting.

Methods: For this study, 33 culture media samples were harvest on day three, from 22 patients undergoing day five embryo transfers. All embryos achieved the blastocyst stage and were split into groups based on their implantation (Negative Implantation, $\mathrm{n}=14$ and Positive Implantation, $n=19)$. The positive implantation cycles resulted in successful ongoing pregnancies. The lipid extraction was performed by the Bligh-Dyer protocol and mass spectra were obtained with a direct infusion into a Q-Tof mass spectrometer. Obtained data was analyzed by Principal Component Analysis (PCA) and Partial Least Square Discrimination Analysis (PLS-DA). The statistical analysis was performed using the Metabo-Analyst 2.0.

Results: The variable importance in the projection (VIP) plot of the PLS-DA provided a list of four ions, in the positive mode, with an area under the curve (AUC) of $73.5 \%$ and eight ions, in the negative model, with and AUC of $72.0 \%$. For both positive and negative modes, possible biomarkers for the negative implantation were identified by the lipidmaps: phosphoethanolamine, dicarboxylic acids, glycerophosphoglycerol, glycerophosphocholine, glicerophosphoinositol, phosphoethanolamine and unsaturated fat acids. The other ions were not identified. These lipids are involved in the GPI anchor biosynthesis and synthesis of lycerophospholipids and phosphate inositol.

Conclusions: MS fingerprinting is useful to identify blastocysts that fail to implant, and therefore this technique could be incorporated in the laboratory routine, adjunct to morphology evaluation to identify embryos that should not be transferred.

\section{0-03. Age male as interference factor in embryo quality. A retrospective study using the technique of "time lapse"}

Guilherme R. F. Rosário', Diana S. Vidal' ${ }^{1}$, Adriana V. Silva ${ }^{1}$, Antônio C. C. Franco ${ }^{1}$

${ }^{1}$ Embryolife Instituto de Medicina Reprodutiva, São José dos Campos/SP.

Objective: Taking into account the timing and the exact time of embryonic cleavages, this study aimed to relate the male age with morfokinects parameters of embryos to D3. Methods: Through a collection system for TimeLapse, traced an ideal curve related to the cleavages of embryos analyzed. The percentage of embryos within the curve was analyzed in correlation with the male age.

Results: The percentage of embryos inside the curve of 
patients aged between 28 and 33 years was $32.6 \%$, 34-39 years was $36.2 \%, 40-45$ years was $41.3 \%$, while that the percentage of embryos inside the curve of patients aged between 46 and 57 years was $26.3 \%$.

Conclusions: We showed decrease percentage of embryos in the optimal curve cleavage to D3 in men aged between 40 and 45 , however there was no significant relation to this event. Further studies should be conducted, evaluating embryos led to the blastocyst stage (D5 or D6).

\section{0-04. Prediction of Metaphase II oocytes according to different serum Anti-Müllerian hormone (AMH) levels in antagonist ICSI cycles}

Joyce B da Silva1, ${ }^{1}$ atiana R Panaino ${ }^{1}$, Maria A Tamm ${ }^{1}$, Paloma Lira ${ }^{1}$, Patricia C F Arêas ${ }^{1}$, Ana C A Mancebo ${ }^{1}$, Marcelo M de Souza ${ }^{1}$, Roberto A Antunes ${ }^{1}$, Maria do Carmo B de Souza ${ }^{1}$

${ }^{1}$ Fertipraxis Centro de Reprodução, Rio de Janeiro, RJ, Brazil

Objective: This paper aims to evaluate the qualitative aspect of ovarian response by metaphase II oocytes according to different serum Anti-Müllerian hormone levels in antagonist ICSI cycles. A prediction index could contribute to the individualization of treatments.

Methods: Observational study, 287 antagonist ICSI cycles in patients who received treatment in a single center, between January 2012 and January 2016. Serum AMH and subgroup analysis were performed according to five $\mathrm{AMH}$ ranges $(\leq 0,3 \mathrm{ng} / \mathrm{mL} ;>0,3$ and $\leq 0,7 \mathrm{ng} / \mathrm{mL} ;>0,7$ and $\leq$ $1,0 \mathrm{ng} / \mathrm{mL} ;>1,0$ and $<3,0 \mathrm{ng} / \mathrm{mL} ; \geq 3,0 \mathrm{ng} / \mathrm{mL}$. Variables were checked as patient's age, serum FSH and antral follicle count at the beginning of the cycle, number of stimulation days, and $\geq 15 \mathrm{~mm}$ on hCG day, number of aspirated oocytes and number of metaphase II oocytes.

Results: $\mathrm{AMH}$ is better in predicting ovarian response to controlled ovarian stimulation than AFC and serum FSH while age is an independent marker. AMH levels $\leq 0,70$ (the worst scenarios) were detected in 140 patients $(48,7 \%)$. This cut-off represented $92 \%$ of the 24 failed cycles (cancelled cycles, no oocytes or immature ones in aspiration)

Conclusions: AMH predicts the quality of ovarian response to stimulation, independently of the patient's age. Women with $\mathrm{AMH}$ levels $\geq 1,0$ e $\leq 3,0 \mathrm{ng} / \mathrm{mL}$ are probably normal responders and a good prognosis may be anticipated Clinical application depends on individual centers examining their own data, correlating $\mathrm{AMH}$ value and the ultimate ovarian response, that is, metaphase II oocytes.

\section{0-05. Evaluation of effects of maintenance and transport in dry ice on the parameters of samples enabling cryopreserved}

\author{
David Til ${ }^{1}$, Vera L L Amaral' ${ }^{1}$, Rafael A Salvador ${ }^{1}$, \\ Alfred Senn'2, Thais S de Paula ${ }^{3}$
}

${ }^{1}$ Universidade do Vale do Itajaí - UNIVALI - Itajaí/SC, Brazil
${ }^{2}$ Fondation pour I'Andrologie, la Biologie et I'Endocrinologie de la Reproduction - FABER - Lausanne, Switzerland ${ }^{3}$ Instituto Sapientiae - São Paulo/SP - Brazil

Objective: Test the effect of dry ice transport of cryopreserved semen samples on the sperm viability.

Methods: 20 normozoospermic samples were cryopreserved, which were then divided into five groups: Group 1 , remained immersed in liquid nitrogen throughout the experiment, inside cryogenic storage tanks. Group 2, cryopreserved straws were transferred into a styrofoam box containing dry ice and kept under these conditions for 48 hours. Group 3, was kept for 48 hours in dry ice, under the same conditions as Group 2, but then returned to the liquid nitrogen. Group 4, was also kept for 48 hours on dry ice, but the styrofoam box containing the samples was in fact sent by airplane, to evaluate the effects of shipping. Group 5, was shipped together with the group 4, but returned to liquid nitrogen after 48 hours on dry ice storage. After thawing the sperm parameters were analyzed by testing their viability, vitality and motility, as well as the mitochondrial activity of the spermatozoa.

Results: A significant decrease in motility recovery rates $(P=0.01)$ and vitality $(P=0.001)$ was observed in all groups tested, compared to the control group. Mitochondrial activity was significantly lower only in Group $5(P=0.04)$, in which the number of sperm cells not stained by the reagent 3,3'-diaminobenzidine was increased.

Conclusions: The transport itself is not a factor that affects the quality of a cryopreserved sample, but the use of dry ice as a method to preserve the samples during transport, was detrimental for the evaluated sperm parameters

\section{0-06. Live births after polar body biopsy and frozen-thawed clea- vage stage embryo transfer: case report}

Fernando Guimarães ${ }^{1}$, Matheus Roque ${ }^{1,2}$, Marcello Valle $^{1}$, Alessandra Kostolias ${ }^{1}$, Rodrigo A de Azevedo ${ }^{1}$, Ciro D Martinhago ${ }^{3}$, Marcos Sampaio ${ }^{4}$, Selmo Geber ${ }^{2,4}$

${ }^{1}$ ORIGEN - Center for Reproductive Medicine, Rio de Janeiro, Brazil

${ }^{2} U F M G$ - Universidade Federal de Minas Gerais, Belo Horizonte, Brazil

${ }^{3}$ Chromosome Genomic Medicine, São Paulo, Brazil

${ }^{4}$ ORIGEN - Center for Reproductive Medicine, Belo Horizonte, Brazil

Introduction: Pre-implantation genetic diagnosis (PGD) or screening (PGS) technology, has emerged and developed in the past few years benefiting couples as it allows the selection and transfer of healthy embryos during IVF treatments. These techniques can be performed in oocytes (polar-body biopsy) or embryos (blastomere or trophectoderm biopsy).

Case report: In this case report, we described the first two live births to be published in Brazil after a polar-body (PB) biopsy. In case 1, a 42-year-old was submitted to PB biopsy with PGS due to the advanced maternal age and poor ovarian reserve. Five MII oocytes underwent first and second polar body biopsy and four cleavage embryos were cryopreserved. The PGS analysis resulted in two euploid embryos (next generation sequency). A frozen-thawed embryo transfer (FET) was performed after endometrial 
priming and a healthy baby was delivered after a cesarean section ( 37 weeks, female, $3390 \mathrm{~g}, 47.5 \mathrm{~cm}$ ). In case 2 , a 40-year old patient with balanced translocation and poor ovarian response was submitted to PB biopsy. Two MII oocytes underwent first and second polar body biopsy and two embryos were cryopreserved in cleavage stage. The analysis resulted in one euploid embryo that was transferred after endometrial priming. A preterm healthy baby (34 weeks, female, $2100 \mathrm{~g}, 40 \mathrm{~cm}$ ) was delivered via cesarean section.

Comments: In conclusion, although the blastocyst biopsy is the norm when performing PGS / PGD during IVF treatments, other alternatives (as PB biopsy) should be taken in account in some specific situations.

\section{0-07. Zika virus and Assisted Reproductive Techniques: to test or not to test, that is the question. Unnecessary costs? Two months post mandatory evaluation in an outbreak area, Rio de Janeiro, Brazil.}

Maria do Carmo B. de Souza ${ }^{1}$, Veronica Raupp ${ }^{2}$, Fernanda Sobrinho ${ }^{2}$, Mariana Menezes ${ }^{2}$, Tatiana R Panaino ${ }^{1}$, Maria A Tamm ${ }^{1}$, Ana C A Mancebo ${ }^{1}$, Ana L R Costa $^{1}$, Roberto A Antunes ${ }^{1}$

${ }^{1}$ Fertipraxis Centro de Reprodução Humana, Rio de Janeiro/RJ, Brazil

${ }^{2}$ Hospital Federal da Lagoa. Rio de Janeiro/RJ, Brazil

Objective: The Zika virus disease is a Public Health Emergency of International Concern by the World Health Organization. Last March 30, 2016, the National Surveillance Agency from Brazil issued Resolucao de Diretoria Colegiada-72, imposing a mandatory testing for ZKV previous to procedures involving Bank Cells and Germinal Tissues. This paper aims at a critical evaluation of this Resolution, from the point of view of evidence and cost - benefits in its first two months.

Methods: The Clinic staff gathered in tasks to accomplish the new rules, checking laboratories in the city to perform the tests, delivery time, costs, refund by health plans, updating data, contacting patients, decision-making in ongoing cases, deadlines for implementation, exchanging ideas among clinics, gathering information from guidelines being built. It was planned a SWOT analysis.

Results: After two months we had 152 tests in 5 clinical situations: one previously symptomatic woman, PCR negative before entering cycle; 2 asymptomatic women IgM positive ( $1,3 \%)$, PCR negative in 25 and 60 days; one husband enrolled as suspect had negative PCR-RT in 13 days and another suspected did not test; a couple only cryopreserved oocytes because the husband's result was not available on the pick-up day. The mean cost of US $\$ 200$ per couple is equivalent to 1,2 day of stimulation protocol. The Clinic staff team won in efficiency and showed fast ability to respond to increased requirements, but testing did not yield security to patients and highed costs.

Conclusions: It is time to review testing before ART in asymptomatic patients. 